\title{
STABILITY OF A PAIR OF FIRST ORDER LINEAR DIFFERENTIAL EQUATIONS
}

\author{
ALAN BRACE and D. E. DAYKIN
}

(Received 12 February 1968)

\section{Introduction}

Consider the simultaneous real differential equations

$$
\dot{x}=a x+b y \text { and } \dot{y}=c x+d y,
$$

where the dot indicates differentiation with respect to time, and $a, b, c, d$ are functions about which the only thing that we know is that they are uniformly bounded,

$$
p_{1} \leqq a \leqq q_{1} ; \quad p_{2} \leqq b \leqq q_{2} ; \quad p_{3} \leqq c \leqq q_{3} ; \quad p_{4} \leqq d \leqq q_{4} .
$$

We say that the bounds (2) have the $\mathscr{S}$-property when they alone imply that the solutions to (1) are uniformly asymptotically stable in the large at the origin.

In this paper we solve completely the problem 'When do the bounds (2) have the $\mathscr{S}$-property?' For particular values of $p_{i}, q_{i}$ they have the $S$-property if every solution eventually arrives at the origin, no matter where it starts on the $x, y$ plane or how the functions $a, b, c, d$ vary within their bounds. On the other hand if we can find some $a, b, c, d$ for which a solution tends to infinity, or goes round a closed path, or is stationary at $\boldsymbol{x} \neq \mathbf{0}$, then the bounds do not have the $\mathscr{S}$-property, even though other solutions may tend to the origin.

Our methods deal with most sets of values of the $p_{i}, q_{i}$ very easily. However in table 4 we give examples of non-trivial sets, which were chosen to illustrate the various possible outcomes of our tests. For those who only wish to use the results, we describe in the last section how this can be done without reading the whole paper.

Some of the methods used here were introduced in [1]. The case where $a=0, b=1, c=-\alpha \beta, d=-(\alpha+\beta)$, and the latent roots $-\alpha,-\beta$ are bounded was solved in [2].

\section{Velocity vector points inwards}

We start by discussing a simple case which introduces something which we will frequently use, namely the minimum value, determined 
from (1) and (2), of the angle between the position vector $\boldsymbol{x}=(x, y)$ and the velocity vector $\dot{\boldsymbol{x}}=(\dot{x}, \dot{y})$. This concept will lead us to what we later call 'centrifugal contours', which remind one of Lyapunov functions, but in some of our cases they are essentially different.

When we say that the velocity vector points inwards we mean that $\dot{\boldsymbol{x}}$ makes an oblique angle with $\boldsymbol{x}$, and so is reducing the distance from the origin. Thus if there is a real number $\rho$ such that $\dot{\boldsymbol{x}} \cdot \boldsymbol{x} /|\dot{\boldsymbol{x}}||\boldsymbol{x}|<\rho<0$ for all $x \neq 0$, and $a, b, c, d$ satisfying (2) then every solution to (1) will tend to $\mathbf{0}$. To prove this by Lyapunov's second method simply consider the function $V=x^{2}+y^{2}$. The above facts give us:

THEOREM 1. The bounds have the $\mathscr{S}$-property if $q_{1}, q_{4}<0$ and

$$
4 q_{1} q_{4}>\left(p_{2}+p_{3}\right)^{2},\left(q_{2}+q_{3}\right)^{2} .
$$

Proof. From (1) and (2) we have

$$
\begin{aligned}
\dot{\boldsymbol{x}} \cdot \boldsymbol{x} & =a x^{2}+(b+c) x y+d y^{2} \\
& \leqq q_{1} x^{2}+(b+c) x y+q_{4} y^{2} \\
& \leqq\left\{\begin{array}{lll}
q_{1} x^{2}+\left(q_{2}+q_{3}\right) x y+q_{4} y^{2} & \text { if } & x y \geqq 0, \\
q_{1} x^{2}+\left(p_{2}+p_{3}\right) x y+q_{4} y^{2} & \text { if } \quad x y \leqq 0,
\end{array}\right.
\end{aligned}
$$

and the result follows.

In exactly the same way, only by having $\dot{\boldsymbol{x}}$ make an acute angle with $\boldsymbol{x}$, we get

THEOREM 2. Every solution to (1) tends to infinity if $p_{1}, p_{4}>0$ and

$$
4 p_{1} p_{4}>\left(p_{2}+p_{3}\right)^{2},\left(q_{2}+q_{3}\right)^{2} \text {. }
$$

The results of this section are independent of the general study in the rest of the paper, but they may well save work in practice.

\section{Latent roots}

It is well known that, when $a, b, c, d$ are constants, the solutions to (1) are stable at $\boldsymbol{x}=\mathbf{0}$ iff the real parts of the latent roots

$$
\frac{1}{2}(d+a) \pm \frac{1}{2} \sqrt{ }\left\{(d+a)^{2}-4(a d-b c)\right\}
$$

of the coefficient matrix

$$
\left(\begin{array}{ll}
a & b \\
c & d
\end{array}\right)
$$

are both strictly negative. Consideration of this fact leads to theorem 3 below.

We let $T$ be the set of 16 numbers obtained by replacing $a$ by $p_{1}$ and by $q_{1}$, by replacing $b$ by $p_{2}$ and by $q_{2}$, and so on, in $a d-b c$. Then we have 
THEOREM 3. The bounds do not have the $\mathscr{S}$-property if $q_{1}+q_{4} \geqq 0$, or if the numbers in the set $T$ are not all strictly positive.

From now on we will assume that the cases dealt with by the theorem have been eliminated. Notice that this means that no solution to (1) and (2) can come to rest at a point $\boldsymbol{x} \neq \mathbf{0}$, or go to infinity along a straight line through the origin.

\section{The $X$ and $Y$ boxes}

On the $x, y$ plane, see for example figure 1 , we draw the box with corners $X$ box: $\left(-q_{2}, p_{1}\right),\left(-p_{2}, p_{1}\right),\left(-p_{2}, q_{1}\right),\left(-q_{2}, q_{1}\right)$.

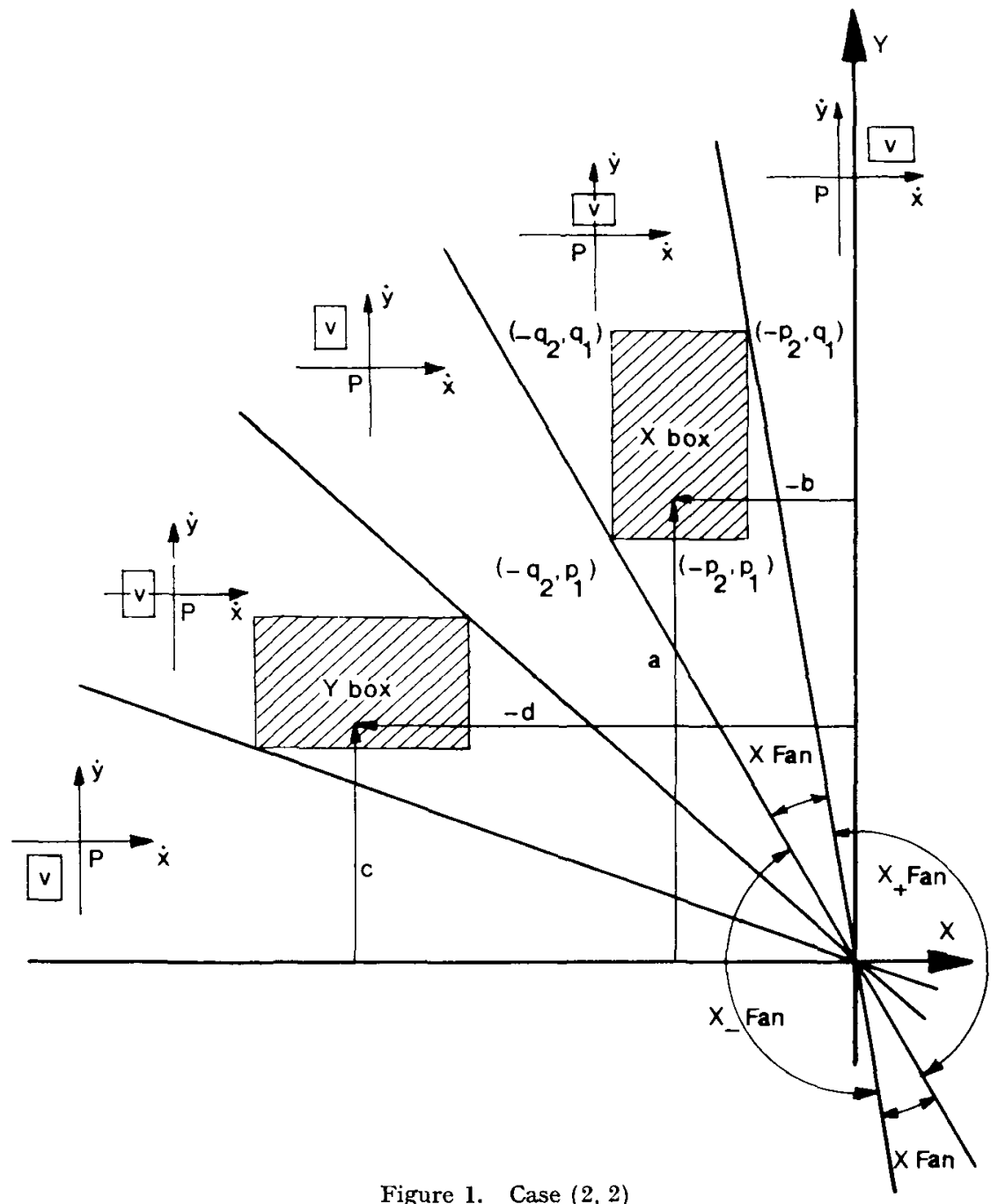


We call this box the $X$ box because it is relevant to the equation $\dot{x}=a x+b y$. The pencil of lines which pass through the origin and the $X$ box and go to infinity in both directions, we call the $X$ fan. The $X$ box includes its boundaries and so does the $X$ fan. The two regions of the plane lying outside the $X$ fan we call the $X_{+}$fan and the $X_{-}$fan respectively, the $X_{+}$fan being the one lying clockwise from the $X$ box. The reason for the notation is that $\dot{x}>0$ for all points $\boldsymbol{x}$ in the $X_{+}$fan, that $\dot{x}<0$ in the $X_{-}$fan, whilst $\dot{x}$ may be positive, zero, or negative in the $X$ fan. To see this consider when $\dot{x}=0$.

In exactly the same way we have the $Y$ box with vertices $Y$ box: $\left(-q_{4}, p_{3}\right),\left(-p_{4}, p_{3}\right),\left(-p_{4}, q_{3}\right),\left(-q_{4}, q_{3}\right)$, and the $Y, Y_{+}, Y_{-}$fans. $W$ e then have a corollary to theorem 3.

COROLlaRY 1. For it to be possible for the bounds to have the $\mathscr{S}$-property we must have that (i) neither the $X$ box nor the $Y$ box contains or touches the origin, and (ii) the $X$ box is properly contained in the $Y_{+}$fan.

\section{Central symmetry and magnification}

We make two observations. Firstly our problem has central symmetry, and so we perform most of our calculations only for points $(x, y)$ in the first two quadrants. The results for the other quadrants follow by symmetry through the origin. Secondly, the state of affairs at any point $P$ is a magnification of the state of affairs at any other point on the line $O P$, for under the transformation $x \rightarrow \omega x, y \rightarrow \omega y$ the velocity $\dot{\boldsymbol{x}}$ is multiplied by $\omega$ but its direction remains unchanged.

\section{The velocity box}

Let $\dot{x}_{i j}$ and $\dot{y}_{i j}$ be the bounds for $\dot{x}$ and $\dot{y}$ determined from (1) and (2), then they are as follows

$$
\begin{gathered}
(x, y) \text { in } 2 \text { nd Quadrant } \\
\dot{x}_{21}=q_{1} x+p_{2} y \leqq \dot{x} \leqq p_{1} x+q_{2} y=\dot{x}_{22} \\
\dot{y}_{21}=q_{3} x+p_{4} y \leqq \dot{y} \leqq p_{3} x+q_{4} y=\dot{y}_{22} \\
(x, y) \text { in } 1 \text { st Quadrant } \\
\dot{x}_{11}=p_{1} x+p_{2} y \leqq \dot{x} \leqq q_{1} x+q_{2} y=\dot{x}_{12} \\
\dot{y}_{11}=p_{3} x+p_{4} y \leqq \dot{y} \leqq q_{3} x+q_{4} y=\dot{y}_{12}
\end{gathered}
$$

Notice that when $(x, y)$ lies on the positive $y$ axis we can use the bounds for either the 1st or 2nd quadrant.

At any point $P=(x, y)$ of the $x, y$ plane we set up velocity axes $P \dot{x}, P \dot{y}$ 
parallel to $O x, O y$. Please refer to figure 1 again. It can be seen from (3) and the central symmetry that for any values of $a, b, c, d$ satisfying (2), the velocity vector $\dot{\boldsymbol{x}}$ for a solution to (1) instantaneously at $P$ must lie in a box drawn relative to the $P \dot{x}, P \dot{y}$ axes. We call this box the velocity $b o x$, or briefly $V$ box. By definition of the $X, X_{+}, X_{-}, Y, Y_{+}, Y_{-}$fans, for any point $P$ of the plane, we can tell immediately the signs of $\dot{x}$ and $\dot{y}$, and hence we can sketch the location of the $V$ box, see figures 1,2 . Note that one side of the $V$ box lies on the $\dot{x}$ or $\dot{y}$ axis only for points $P$ on the edges of the $X$ and $Y$ fans. Also the $V$ box cannot contain or touch the origin $P$, for this would give a stationary solution. The coordinates $(\dot{x}, \dot{y})$ of the corners of the $V$ box are as follows:

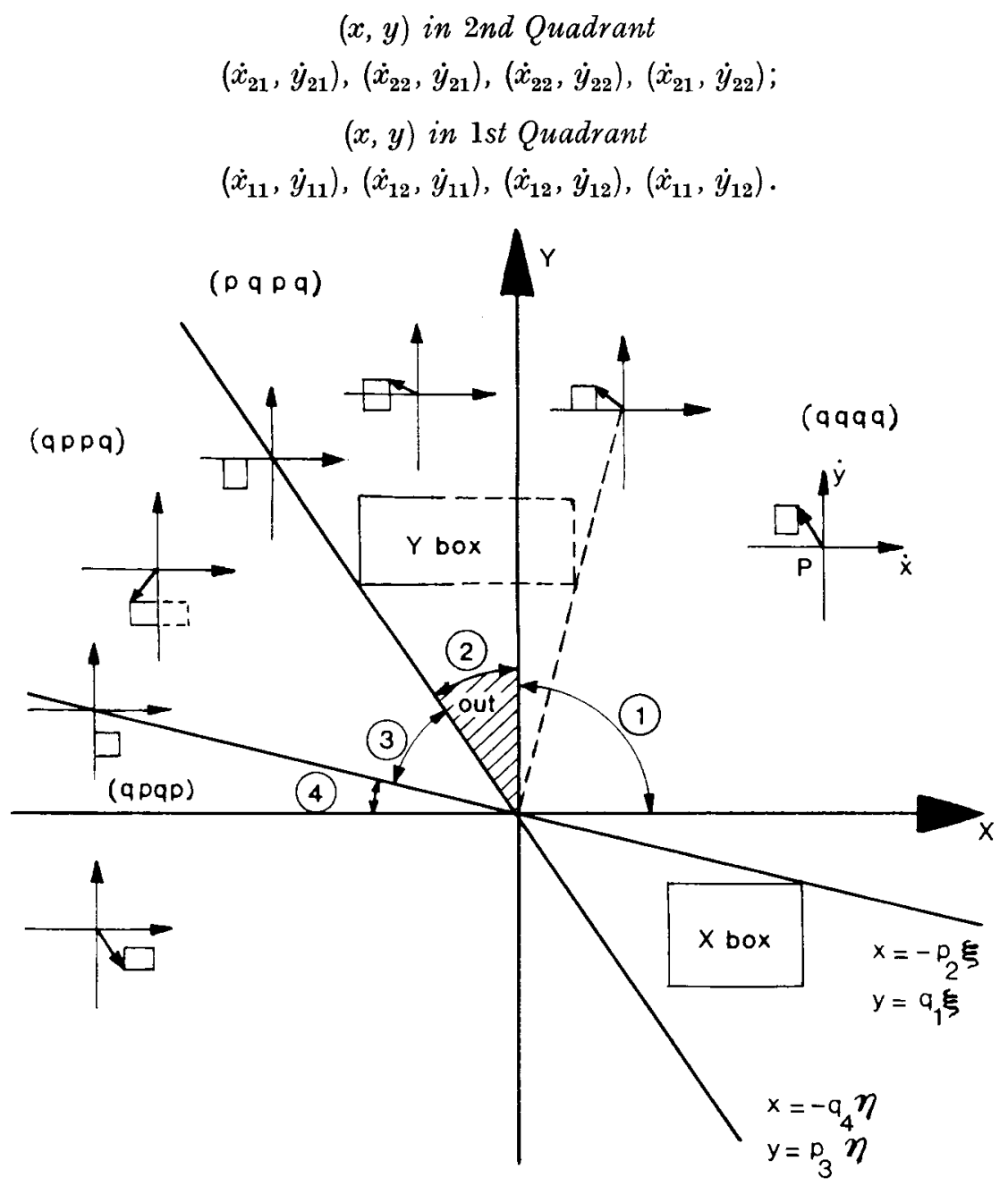

Figure 2. Cases $\left(4, \frac{1}{2}\right)$ and $(4,2)$ 
It is necessary for us to study the way that the $V$ box changes as $P$ varies over the plane, but in view of the properties mentioned in Section 5, we may imagine $P$ to move in a circle.

\section{Motion of the $V$ box}

For any point $P=(x, y)$ of the $x, y$ plane, the coordinates $\left(\dot{x}_{G}, \dot{y}_{G}\right)$, relative to the $P \dot{x}, P \dot{y}$ velocity axes, of the centre of gravity $G$ of the $V$ box at $P$ are obtained by taking the mean values of $a, b, c, d$ in (2). Hence they are

$$
\begin{aligned}
& \dot{x}_{G}=\frac{1}{2}\left(p_{1}+q_{1}\right) x+\frac{1}{2}\left(p_{2}+q_{2}\right) y, \\
& \dot{y}_{G}=\frac{1}{2}\left(p_{3}+q_{3}\right) x+\frac{1}{2}\left(p_{4}+q_{4}\right) y,
\end{aligned}
$$

and by our assumption in section 3 they are not zero.

Next we transform to polar coordinates, $x=r \cos \theta, y=r \sin \theta$. We let $\lambda, \alpha$ and $\mu, \beta$ be the polar coordinates of the centres of gravity of the $X$ and $Y$ boxes respectively, so that $\lambda, \mu>0$ and

$$
\begin{array}{ll}
\lambda \cos \alpha=-\frac{1}{2}\left(p_{2}+q_{2}\right) ; & \lambda \sin \alpha=\frac{1}{2}\left(p_{1}+q_{1}\right), \\
\mu \cos \beta=-\frac{1}{2}\left(p_{4}+q_{4}\right) ; & \mu \sin \beta=\frac{1}{2}\left(p_{3}+q_{3}\right) .
\end{array}
$$

Equations (4) then become

$$
\begin{aligned}
& \dot{x}_{G}=r \lambda \sin (\alpha-\theta) \\
& \dot{y}_{G}=r \mu \sin (\beta-\theta),
\end{aligned}
$$

and we are in a position to prove

Lemma 1. Given $r>0$, there is a non-degenerate ellipse, fixed in the velocity axes, such that as $P$ moves once round the circle of radius $r$ centred at 0 , the centroid $G$ moves once round the ellipse. The directions of turning are the same if $\sin (\beta-\alpha)>0$ but opposite if $\sin (\beta-\alpha)<0$. Further in half a revolution of $P$, the points $O, P, G$ are colinear, or more importantly the $V$ box completely crosses $O P$, at most twice.

Proof. Equations (5) show that $\dot{x}_{G}$ and $\dot{y}_{G}$ are bounded. Eliminating $\theta$ between these equations gives a second order equation in $\dot{x}_{G}, \dot{y}_{G}$, which must therefore be the equation of an ellipse. The ellipse cannot be degenerate because then $G$ would pass through $P$.

Putting $\tan \phi=\dot{y}_{G} / \dot{x}_{G}$ and differentiating gives

$$
\dot{\phi}=\dot{\theta}(\mu / \lambda)[\{\cos \phi\} / \sin (\alpha-\theta)]^{2} \sin (\beta-\alpha) \text { and } \lambda, \mu>0,
$$

so the part of the lemma about the direction of turning follows immediately. Notice that we cannot have $\sin (\beta-\alpha)=0$ for then the $X$ fan touches the $Y$ fan. 
The condition that $O, P, G$ be colinear yields the equation

$$
\lambda \cos \alpha \tan ^{2} \theta-(\mu \cos \beta+\lambda \sin \alpha) \tan \theta+\mu \sin \beta=0,
$$

which has at most two roots $\theta$ in a range of $\pi$ for $\theta$. Thus $G$ either crosses $O P$ twice, or touches $O P$ once, or does not touch $O P$ as $\theta$ runs through $\pi$, a half revolution for $P$. This completes the proof of the lemma.

We note in passing that for $a, b, c, d$ constant, the remarks in the above lemma still apply. We merely put $q_{1}=a=p_{1}$, etc. Thus in this constant case the velocity vector either crosses the radius vector twice, or touches it once, or stays clear of it, as the radius vector rotates through an angle $\pi$. The lines where it crosses or touches will be given by the roots of the equation

$$
c x^{2}+(d-a) x y-b y^{2}=0 .
$$

\section{Transformations}

Under the transformation $A$ defined by $x=-x^{\prime}, y=y^{\prime}$ the pair of equations

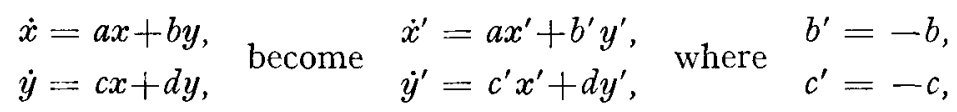

and if one pair is stable so is the other. The change in sign of $b$ and $c$ must cause a change in the bounds, namely $p_{2}^{\prime}=-q_{2}, p_{3}^{\prime}=-q_{3}, q_{2}^{\prime}=-p_{2}$, $q_{3}^{\prime}=-p_{3}$. This change shows that if we reflect the $X$ box in the $y$ axis we get the $X^{\prime}$ box, and if we reflect the $Y$ box in the $x$ axis we get the $Y^{\prime}$ box. Using these facts we can transform certain cases of our equations into other cases. We will use $A$ and another transformation $B$. The two, together with their effects, are:

A: $x=-x^{\prime}, y=y^{\prime}\left\{\begin{array}{l}X^{\prime} \text { is } X \text { reflected in the } y \text { axis, } \\ Y^{\prime} \text { is } Y \text { reflected in the } x \text { axis, }\end{array}\right.$

$$
\begin{aligned}
p_{1} \rightarrow p_{1}^{\prime} & -q_{2} \rightarrow p_{2}^{\prime} & -q_{3} \rightarrow p_{3}^{\prime} & p_{4} \rightarrow p_{4}^{\prime} \\
q_{1} \rightarrow q_{1}^{\prime} & -p_{2} \rightarrow q_{2}^{\prime} & -p_{3} \rightarrow q_{3}^{\prime} & q_{4} \rightarrow q_{4}^{\prime}
\end{aligned}
$$

B: $x=y^{\prime}, y=-x^{\prime}\left\{\begin{array}{l}X^{\prime} \text { is } Y \text { rotated through }-\frac{1}{2} \pi \\ Y^{\prime} \text { is } X \text { rotated through }+\frac{1}{2} \pi .\end{array}\right.$

$$
\begin{array}{rlll}
p_{4} \rightarrow p_{1}^{\prime} & -q_{3} \rightarrow p_{2}^{\prime} & -q_{2} \rightarrow p_{3}^{\prime} & p_{1} \rightarrow p_{4}^{\prime} \\
q_{4} \rightarrow q_{1}^{\prime} & -p_{3} \rightarrow q_{2}^{\prime} & -p_{2} \rightarrow q_{3}^{\prime} & q_{1} \rightarrow q_{4}^{\prime}
\end{array} .
$$

Notice that the set $T$ is unchanged by $A$ or $B$.

The $X$ and $Y$ boxes may lie anywhere on the plane, except of course 
that the cases dealt with by theorem 3 have been omitted. In order to describe the remaining cases we adopt the following notation. We write 2 to denote that a box is in the second quadrant, we write $\frac{2}{3}$ to denote it straddles the negative $x$ axis, and so on, and in (3.1), the first number ' 3 ' refers to the $X$ box, the second number ' 1 ' refers to the $Y$ box. We have 17 cases to deal with and they are listed in Table 1 . Transformations reduce these 17 to 6 basic cases, but no further reduction is possible. We will show that the bounds have the $\mathscr{S}$-property in 1 of the 6 cases. That leaves just 5 basic cases, for each of which the bounds may or may not have the $\mathscr{S}$ property, depending on the actual numerical values of the $p$ 's and $q$ 's. Given the $p$ 's and $q$ 's whether the $\mathscr{S}$-property holds can be tested simply by adding up some numbers in a way which we will describe later.

TABLE 1

\begin{tabular}{lllll}
\hline Case & Instructions & Case & \multicolumn{1}{l}{ Instructions } & \\
\hline$(3,1)$ & $\mathscr{S}$ & $\left(3, \frac{3}{4}\right)$ & $A \rightarrow\left(4, \frac{1}{2}\right)$ & \\
$\left(\frac{3}{4}, 1\right)$ & theorem 4, table 3 & $(2,4)$ & $A \rightarrow(1,1)$ & $B \rightarrow(4,2)$ \\
$(4,1)$ & theorem 4, table 3 & $\left(\frac{2}{3}, 4\right)$ & $A \rightarrow\left(\frac{1}{4}, 1\right)$ & $B \rightarrow\left(4, \frac{1}{2}\right)$ \\
$(1,1)$ & $B \rightarrow\left(4, \frac{1}{2}\right)$ & $(3,4)$ & $A \rightarrow(4,1)$ & \\
$(1,1)$ & $B \rightarrow(4,2)$ & $\left(\frac{3}{4}, 4\right)$ & $A \rightarrow\left(\frac{3}{4}, 1\right)$ & \\
$\left(4, \frac{1}{2}\right)$ & table 2 & $(4,4)$ & $A \rightarrow(3,1)$ & $\mathscr{S}$ \\
$(4,2)$ & table 2 & $\left(3, \frac{1}{4}\right)$ & $A \rightarrow\left(4, \frac{1}{4}\right)$ & $B \rightarrow\left(\frac{3}{4}, 1\right)$ \\
$(3,3)$ & $A \rightarrow(4,2)$ & $\left(\frac{3}{4}, 1\right)$ & special & \\
& & $\left(4, \frac{1}{4}\right)$ & & $B \rightarrow\left(\frac{3}{4}, 1\right)$ \\
\hline
\end{tabular}

\section{The constant case}

Without preamble we say that we will need the solutions to (1) for the case in which $a, b, c, d$ are all constants. Putting $s=(d-a)^{2}+4 b c$ the solution is

$$
\frac{1}{2} \log k\left|c x^{2}+(d-a) x y-b y^{2}\right|= \begin{cases}\frac{(d+a) x}{2 b y-(d-a) x} & \text { if } s=0, \\ \frac{(d+a)}{\sqrt{ }-s} \arctan \left(\frac{(d-a) x-2 b y}{x \sqrt{ }-s}\right) & \text { if } s<0,\end{cases}
$$

where $k$ is the constant of integration, and the range for $\arctan$ is $-\frac{1}{2} \pi$ to $+\frac{1}{2} \pi$. Please observe the moduli inside the logarithms.

When $s>0$ the solutions either look like hyperbolae and have two asymptotes, or they look figures of 8 which have had their tops and bottoms pulled to infinity along a single asymptote. When $s=0$ the solutions look 
like the figure $S$ with its ends pulled to infinity along a single asymptote. Finally when $s<0$ the solutions are spirals, a special case of which is the ellipse. We only mention these facts as a matter of interest. All that we will need will be finite parts of the curves.

\section{The basic cases}

Cases $\left(4, \frac{1}{2}\right)$ and $(4,2)$. Please refer to figure 2 where we have shown the more important characteristics of these cases. In both of them the $X$ box lies in the 4th quadrant. The $Y$ box is either in the 2nd quadrant or straddling the positive $y$ axis, so we have drawn one end of the $Y$ box dotted to indicate the two possibilities. The mathematical analysis is the same for both.

TABLE 2: Cases $\left(4, \frac{1}{2}\right)$ and $(4,2)$

$$
\begin{gathered}
s_{1}=\left(q_{4}-q_{1}\right)^{2}+4 q_{2} q_{3} \quad s_{3}=\left(q_{4}-q_{1}\right)^{2}+4 p_{2} p_{3} \\
s_{2}=\left(q_{4}-p_{1}\right)^{2}+4 q_{2} p_{3} \quad s_{4}=\left(p_{4}-q_{1}\right)^{2}+4 p_{2} q_{3} \\
\frac{1}{2} \log \left|\frac{q_{1} p_{4}-p_{2} q_{3}}{p_{1} q_{4}-q_{2} p_{3}}\right|
\end{gathered}
$$

\begin{tabular}{lcl}
\hline$s_{i}<0$ & $s_{i}=0$ & $s_{i}>0$ \\
\hline$\frac{q_{1}+q_{4}}{\sqrt{ }-s_{1}}\left[\frac{\pi}{2}-\arctan \frac{q_{4}-q_{1}}{\sqrt{ }-s_{1}}\right]$ & $\frac{q_{4}+q_{1}}{q_{4}-q_{1}}$ & $\frac{q_{1}+q_{4}}{2 \sqrt{ } s_{1}} \log \left|\frac{q_{1}-q_{4}-\sqrt{ } s_{1}}{q_{1}-q_{4}+\sqrt{ } s_{1}}\right|$ \\
$\frac{p_{1}+q_{4}}{\sqrt{ }-s_{2}}\left[\frac{\pi}{2}+\arctan \frac{s_{2}+q_{4}^{2}-p_{1}^{2}}{2 q_{4} \sqrt{ }-s_{2}}\right]$ & $\frac{2 q_{4}}{p_{1}-q_{4}}$ & $\frac{p_{1}+q_{4}}{2 \sqrt{ } s_{2}} \log \left|\frac{p_{1}^{2}-\left(q_{4}-\sqrt{ } s_{2}\right)^{2}}{p_{1}^{2}-\left(q_{4}+\sqrt{ } s_{2}\right)^{2}}\right|$ \\
$\frac{q_{1}+q_{4}}{\sqrt{ }-s_{3}}\left[\arctan \frac{q_{1}+q_{4}}{\sqrt{ }-s_{3}}-\arctan \frac{s_{3}+q_{4}^{2}-q_{1}^{2}}{2 q_{4} \sqrt{ }-s_{3}}\right]$ & $\frac{q_{4}+q_{1}}{q_{4}-q_{1}}$ & $\frac{q_{1}+q_{4}}{2 \sqrt{ } s_{3}} \log \left|\frac{q_{1}-q_{4}-\sqrt{ } s_{3}}{q_{1}-q_{4}+\sqrt{ } s_{3}}\right|$ \\
$\frac{q_{1}+p_{4}}{\sqrt{ }-s_{4}}\left[\arctan \frac{p_{4}-q_{1}}{\sqrt{ }-s_{4}}-\arctan \frac{q_{1}+p_{4}}{\sqrt{ }-s_{4}}\right]$ & $\frac{2 q_{1}}{q_{1}-p_{4}}$ & $\frac{q_{1}+p_{4}}{2 \sqrt{ } s_{4}} \log \left|\frac{p_{4}^{2}-\left(q_{1}+\sqrt{ } s_{4}\right)^{2}}{p_{4}^{2}-\left(q_{1}-\sqrt{ } s_{4}\right)^{2}}\right|$ \\
\hline
\end{tabular}

The parametric equation of the line through the origin which touches the top right corner of the $X$ box is $x=-p_{2} \xi, y=q_{1} \xi$. We also need the other line which touches the $Y$ box as shown. These two lines divide the upper half plane into the regions numbered $1,2,3,4$, and we have sketched the location of the $V$ box for representative points of the regions.

For any $\omega>0$, all solutions to (1) and (2) which pass through the point $(\omega, 0)$ are moving up and to the left. We call the one which at all times make as acute an angle as possible with the radius vector the centrifugal (anticlockwise) contour $C_{\omega}$ through $(\omega, 0)$. To find $C_{\omega}$ in the first quadrant we want the velocity vector to always lie at the top right corner of the $V$ box as shown. Let us adopt the convention whereby $(p q p p)$ for example means $a=p_{1}, b=q_{2}, c=p_{3}, d=p_{4}$. Then the inequalities (3) 
show us that the values of $a, b, c, d$ which give $C_{\omega}$ for region 1 are $(q q q q)$. In particular $a, b, c, d$ are constants and so we can find the equation of $C_{\omega}$ from (7).

We need to follow this upper right corner of the $V$ box to get $C_{\omega}$ for region 2 , but the bounds (3) show that we must change the values of (abcd) to $(p q p q)$. There is no danger that $C_{\omega}$ will go to the origin before reaching the negative $x$ axis, for this would require the $V$ box to cross $O P$ between $O$ and $P$, and the diagram reveals that this is not possible. Also a solution could only go straight to infinity in the region labelled 'out', and this possibility was ruled out in section 3 . When $C_{\omega}$ moves into region 3 we want the upper left corner of the $V$ box, so $(a b c d)=(q p p q)$. Similarly in region 4 we have $(a b c d)=(q p q p)$, and $C_{\omega}$ meets the negative $x$ axis at a finite point $(-\delta, 0)$. By virtue of the remarks of section 5 , if $\delta>\omega$ then $C_{\omega}$ spirals away to infinity as it goes round and round the origin. If $\delta=\omega$ then $C_{\omega}$ moves round the origin in a fixed orbit. Finally we assert that if $\delta<\omega$ then the bounds have the $\mathscr{S}$-property. For suppose we are at any point $P$ of the plane. A centrifugal contour $C_{\omega}$ will pass through $P$ for some value of $\omega$. The tangent to $C_{\omega}$ at $P$ is the line from $P$ to a corner of the $V$ box, so all solutions through $P$ either follow $C_{\omega}$ or move to points met by centrifugal contours $C_{\omega^{\prime}}$ with $\omega^{\prime}<\omega$. No solution can cross the positive $x$ axis in a clockwise direction, nor can one come to rest. Since $\delta<\omega$ the curve $C_{\omega}$ spirals in to the origin forcing all solutions to (1) and (2) in with it.

We may assume that $\omega=1$ and write $\delta_{4}$ for the corresponding $\delta$. It then only remains to calculate $\delta_{4}$, for the bounds have the $\mathscr{S}$-property iff $\delta_{4}<1$. We must deal with each of the regions $1,2,3,4$ separately. We write $s_{1}, s_{2}, s_{3}, s_{4}$ for the values of the constant $(d-a)^{2}+4 b c$. Since the $s_{i}$ may be positive, zero or negative, the equation (7) of $C_{1}$ may take three possible forms. Substituting the values of $(a b c d)$ in (7) gives us the four equations for the four regions for $C_{1}$, in terms of the unknown constants of integration $k_{1}, k_{2}, k_{3}, k_{4}$. Now $C_{1}$ goes from $(1,0)$ to $\left(-\delta_{4}, 0\right)$. Let $\left(0, \delta_{1}\right)$, $\left(-q_{4} \delta_{2}, p_{3} \delta_{2}\right)$ and $\left(-p_{2} \delta_{3}, q_{1} \delta_{3}\right)$ be the points at which $C_{1}$ crosses from regions 1 to 2 , from 2 to 3 , and from 3 to 4 respectively. We substitute the two boundary points $(1,0)$ and $\left(0, \delta_{1}\right)$ in the equation for region 1 to obtain two equations. Then we substitute $\left(0, \delta_{1}\right)$ and $\left(-q_{4} \delta_{2}, p_{3} \delta_{2}\right)$ in the equation for region 2 , and similarly for the other two regions. Twice the left hand sides of the eight equations are the eight expressions (i), (ii), $\cdots$, (viii) below.
Region
Trice left hand side
(i) $1\left\{\begin{array}{l}\log k_{1}+\log \left|q_{3}\right| \text {, } \\ \log k_{1}+\log \delta_{1}^{2}+\log \left|q_{2}\right| \text {, }\end{array}\right.$
(iii) $2\left(\log k_{2}+\log \delta_{1}^{2}+\log \left|q_{2}\right|\right.$,

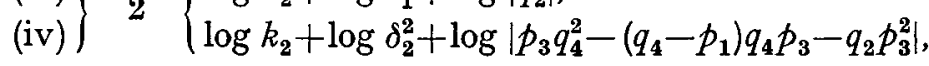


Region Twice left hand side
(v) $3\left\{\log k_{3}+\log \delta_{2}^{2}+\log \left|p_{3} q_{4}^{2}-\left(q_{4}-q_{1}\right) q_{4} p_{3}-p_{2} p_{3}^{2}\right|\right.$,
(vi) $3\left\{\log k_{3}+\log \delta_{3}^{2}+\log \left|p_{3} p_{2}^{2}-\left(q_{4}-q_{1}\right) p_{2} q_{1}-p_{2} q_{1}^{2}\right|\right.$,
$\left.\begin{array}{r}\text { (vii) } \\ \text { (viii) }\end{array}\right\} 4\left\{\begin{array}{l}\log k_{4}+\log \delta_{3}^{2}+\log \left|q_{3} p_{2}^{2}-\left(p_{4}-q_{1}\right) p_{2} q_{1}-p_{2} q_{1}^{2}\right| \text {, } \\ \log k_{4}+\log \delta_{4}^{2}+\log \left|q_{3}\right| .\end{array}\right.$

Forming the sum (viii) $-($ vii $)+($ vi $)-(v)+($ iv $)-($ iii $)+($ ii $)-(i)$ gives us trivially

$$
\left(\log \delta_{4}^{2}\right)-\log \left|\frac{p_{2} q_{3}-q_{1} p_{4}}{p_{1} q_{4}-q_{2} p_{3}}\right| .
$$

Also we can see from (7) that in each of the 24 possible right hand sides the $\delta$ 's cancel out. For each of the 4 regions, and for each of the 3 possible values of $s_{i}$, we evaluate the differences of the right hand sides (remember $s_{i}$ is constant in each region), and this gives us table 2. The table is used as follows. We work out $s_{1}, s_{2}, s_{3}, s_{4}$, then for $i=1,2,3,4$ we take the entry

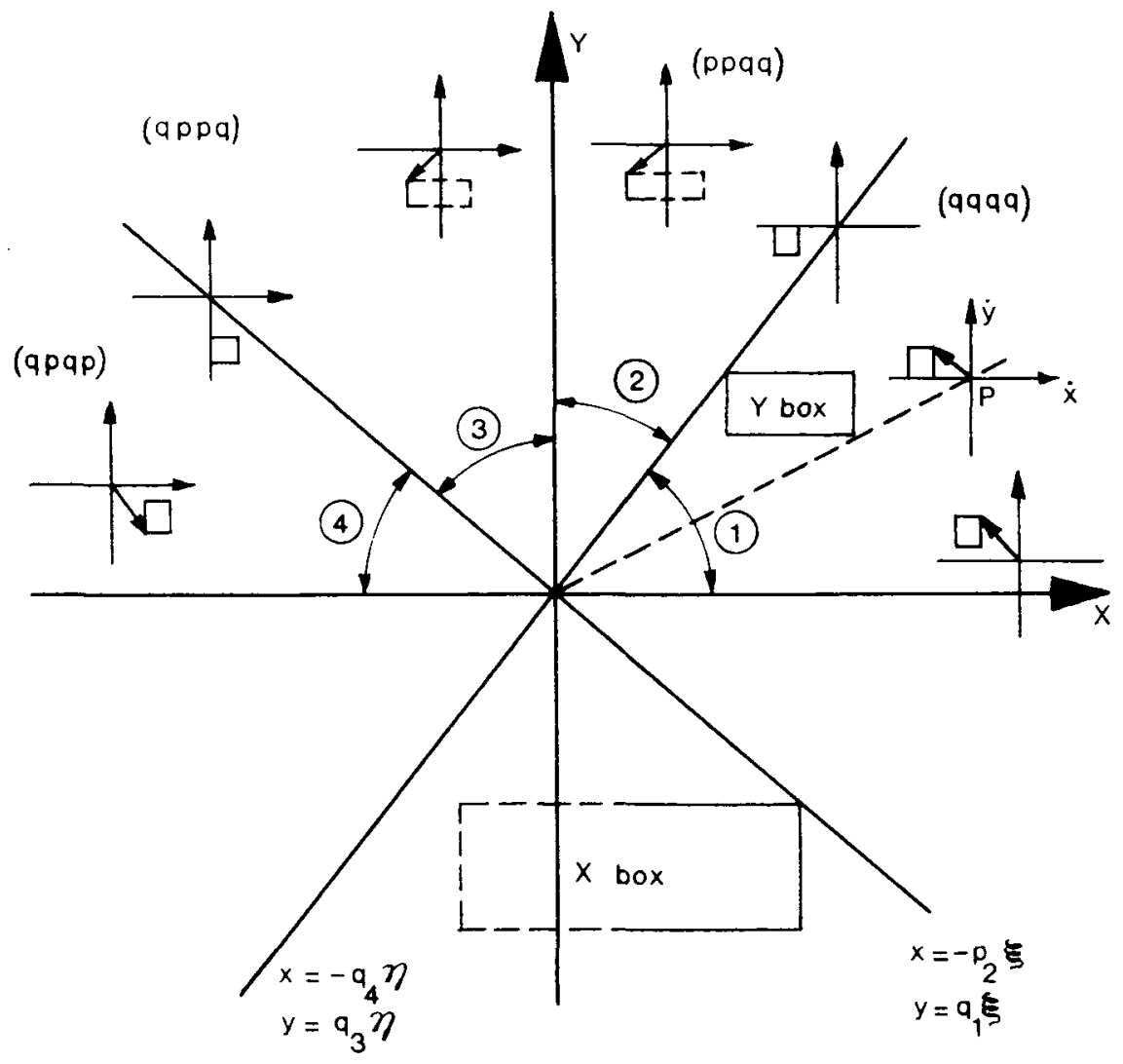

Figure 3. Cases $\left(\frac{3}{4}, 1\right)$ and $(4,1)$ 
from the $i$-th row determined by the sign of $s_{i}$. Finally we add up the values of the four entries and that of the expression at the head of the table, which was obtained from (8). If the result is negative then $\delta_{\mathbf{4}}<1$ and the bounds have the $\mathscr{S}$-property, otherwise they do not.

Cases $\left(\frac{\mathbf{3}}{4}, 1\right)$ and $(4,1)$. In these cases $C_{\omega}$ might come into the origin in less than one revolution, which will happen iff the $V$ box crosses $O P$ completely between $O$ and $P$. We deal with such situations in theorem 4 below.

Theorem 4. In cases $\left(\frac{3}{4}, 1\right)$ and $(4,1)$ if

$$
\begin{array}{ll}
q_{3} x^{2}+\left(q_{4}-p_{1}\right) x y-p_{2} y^{2}=0 & \text { has a positive root } x / y, \\
q_{3} x^{2}+\left(p_{4}-q_{1}\right) x y-p_{2} y^{2}=0 & \text { has a negative root } x / y,
\end{array}
$$

then the bounds have the $\mathscr{S}$-property.

PROoF. If the first condition holds then the top left hand corner of the $V$ box will lie on the radius vector somwehere in the first quadrant, while if the second condition holds then the bottom left hand corner of the $V$ box will lie on the radius vector somewhere in the second quadrant. The $V$ box stands clear of the radius vector on the positive $x$ axis. Therefore the conditions (9) imply that as the radius vector swings through a half revolution anticlockwise from the positive $x$ axis, the $V$ box crosses over the radius vector completely. If it crosses completely once, then by symmetry and Lemma 1 it crosses completely twice. During the first crossing the radius vector is 'catching up' with the $V$ box, while during the second crossing the $V$ box is 'catching up' with the radius vector.

Let $O P_{\mathbf{1}}$ be the line where the first corner of the $V$ box crosses the radius vector and let $O P_{\mathbf{2}}$ be the line where the last corner crosses the radius vector, during the first crossing. Let $P_{1}^{\prime}$ and $P_{2}^{\prime}$ be the central images of $P_{1}$ and $P_{2}$. It follows that no solution to (1) and (2) can move clockwise over $P_{1}^{\prime} O P_{1}$ or anti-clockwise over $P_{2}^{\prime} O P_{2}$. Hence all solutions go to the origin or to one of the fans $P_{1} O P_{2}$ and $P_{1}^{\prime} O P_{2}^{\prime}$. It is easy to see that once a solution moves into one of the fans it must go to the origin. This completes the proof of the theorem.

If neither of the conditions (9) hold then an anti-clockwise centrifugal contour exists and, by analysis similar to the preceding cases, whether the $\mathscr{S}$-property holds can be tested using table $\mathbf{3}$.

Case $(3,1)$. The diagram for this case is very similar to figure 3 , and so is omitted. It is easy to see that the $V$ box always crosses the radius vector completely as it swings through a quarter revolution anticlockwise from the positive $x$ axis. It follows, using the reasoning of theorem 4 , that in this case the bounds always have the $\mathscr{S}$-property. 
TABLE 3: Cases $\left(\frac{3}{4}, 1\right)$ and $(4,1)$

$$
\begin{gathered}
s_{1}=\left(q_{4}-q_{1}\right)^{2}+4 q_{2} q_{3} \quad s_{3}=\left(q_{4}-q_{1}\right)^{2}+4 p_{2} p_{3} \\
s_{2}=\left(q_{4}-p_{1}\right)^{2}+4 p_{2} q_{3} \quad s_{4}=\left(p_{4}-q_{1}\right)^{2}+4 p_{2} q_{3} \\
\frac{1}{2} \log \left|\frac{\left(p_{1} q_{4}-p_{2} q_{3}\right)\left(q_{1} p_{4}-p_{2} q_{3}\right)}{\left(q_{1} q_{4}-q_{2} q_{3}\right)\left(q_{1} q_{4}-p_{2} p_{3}\right)}\right|
\end{gathered}
$$

\begin{tabular}{lcc}
\hline$s_{i}<0$ & $s_{i}=0$ & $s_{i}>0$ \\
\hline$\frac{q_{1}+q_{4}}{\sqrt{ }-s_{1}}\left[\arctan \frac{s_{1}+q_{4}^{2}-q_{1}^{2}}{2 q_{4} \sqrt{ }-s_{1}}-\arctan \frac{q_{4}-q_{1}}{\sqrt{ }-s_{1}}\right]$ & -1 & $\frac{q_{1}+q_{4}}{2 \sqrt{ } s_{1}} \log \left|\frac{q_{1}+q_{4}-\sqrt{ } s_{1}}{q_{1}+q_{4}+\sqrt{ } s_{1}}\right|$ \\
$\frac{p_{1}+q_{4}}{\sqrt{ }-s_{2}}\left[\frac{\pi}{2}-\arctan \frac{s_{2}+q_{4}^{2}-p_{1}^{2}}{2 q_{4} \sqrt{ }-s_{2}}\right]$ & $\frac{2 q_{4}}{q_{4}-p_{1}}$ & $\frac{p_{1}+q_{4}}{2 \sqrt{ } s_{2}} \log \left|\frac{p_{1}^{2}-\left(q_{4}+\sqrt{ } s_{2}\right)^{2}}{p_{1}^{2}-\left(q_{4}-\sqrt{ } s_{2}\right)^{2}}\right|$ \\
$\frac{q_{1}+q_{4}}{\sqrt{ }-s_{3}}\left[\frac{\pi}{2}+\arctan \frac{q_{1}+q_{4}}{\sqrt{ }-s_{3}}\right]$ & -1 & $\frac{q_{1}+q_{4}}{2 \sqrt{ } s_{3}} \log \left|\frac{q_{1}+q_{4}-\sqrt{ } s_{3}}{q_{1}+q_{4}+\sqrt{ } s_{3}}\right|$ \\
$\frac{q_{1}+p_{4}}{\sqrt{-s_{4}}}\left[\arctan \frac{p_{4}-q_{1}}{\sqrt{-}-s_{4}} \arctan \frac{q_{1}+p_{4}}{\sqrt{-s_{4}}}\right]$ & $\frac{2 q_{1}}{q_{1}-p_{4}}$ & $\frac{q_{1}+p_{4}}{2 \sqrt{ } s_{4}} \log \left|\frac{p_{4}^{2}-\left(q_{1}+\sqrt{ } s_{4}\right)^{2}}{p_{4}^{2}-\left(q_{1}-\sqrt{ } s_{4}\right)^{2}}\right|$
\end{tabular}

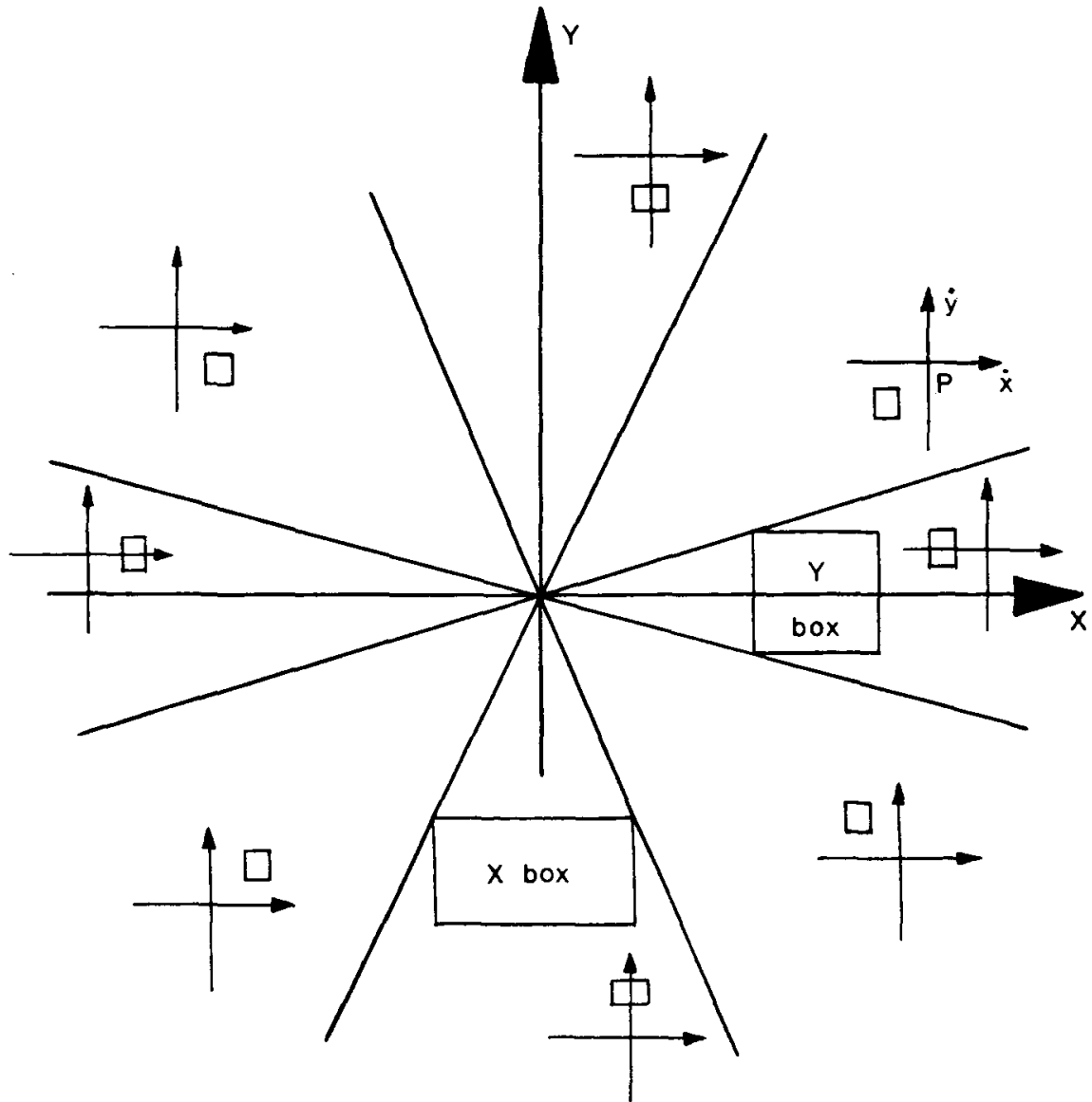

Figure 4. Case $\left(\frac{3}{4}, \frac{1}{4}\right)$ 
Case $\left(\frac{\mathbf{3}}{\mathbf{4}}, \frac{\mathbf{1}}{\mathbf{4}}\right)$. See figure 4 . In all previous cases no solution could move clockwise over the $x$ axis. This case $\left(\frac{3}{4}, \frac{1}{4}\right)$ is special because, as can be seen from figure 4 , it may be possible for solutions to move continuously clockwise or continuously anticlockwise, and there may be both an anti-clockwise and a clockwise centrifugal contour. Solutions may not move continuously anticlockwise iff

$$
\begin{array}{ll}
q_{3} x^{2}+\left(q_{4}-p_{1}\right) x y-p_{2} y^{2}=0 & \text { has a positive root or } \\
q_{3} x^{2}+\left(p_{4}-q_{1}\right) x y-p_{2} y^{2}=0 & \text { has a negative root. }
\end{array}
$$

To see this please refer to the start of the proof of theorem 4. Similarly solutions may not move continuously clockwise iff

$$
\begin{array}{ll}
p_{3} x^{2}+\left(q_{4}-p_{1}\right) x y-q_{2} y^{2}=0 & \text { has a negative root or } \\
p_{3} x^{2}+\left(p_{4}-q_{1}\right) x y-q_{2} y^{2}=0 & \text { has a positive root. }
\end{array}
$$

There are four possibilities:

(i) If solutions cannot move continuously clockwise and cannot move continuously anticlockwise then by reasoning similar to that of theorem 4 , the bounds must have the $\mathscr{S}$-property.

(ii) If solutions may move anticlockwise but not clockwise then an anticlockwise centrifugal contour exists and whether the $\mathscr{S}$-property holds can be tested using table 3 .

(iii) If solutions may move clockwise but not anticlockwise then a clockwise centrifugal contour exists and whether the $\mathscr{S}$-property holds can be tested by applying transformation $A$ and then using table 3 .

(iv) If none of the above three possibilities applies, then the $V$ box must always straddle the radius vector, solutions may move continuously clockwise or anticlockwise, and there are clockwise and anticlockwise centrifugal contours, both of which must be tested, as in (ii) and (iii).

\section{Summary of method}

First try theorems 1, 2, 3. Failing these find your case in table 1, the case notation is introduced at the end of section 8 , and $\mathscr{P}$ (not $\mathscr{S}$ ) mean that the bounds have (do not have) the $\mathscr{S}_{\text {-property. If instructed }}$ to do so change your case by changing your bounds $p_{i}, q_{i}$ to $p_{i}^{\prime}, q_{i}^{\prime}$ by the transformation $A, B$ of section 8 . Then use the table indicated, except that in cases $\left(\frac{3}{4}, 1\right)$ and $(4,1)$ one must first try theorem 4 . To use a table work out $s_{1}, s_{2}, s_{3}, s_{4}$. Then for $i=1,2,3,4$ take the value of the entry in the $i$-th row of the table indicated by the sign of $s_{i}$. Finally add up the four values together with the expression at the head of the table. The bounds have the $\mathscr{S}$-property iff the sum is negative. The special case $\left(\frac{\mathbf{3}}{\mathbf{4}}, \frac{1}{4}\right)$ is explained in the last part of section 10. 
$[15]$

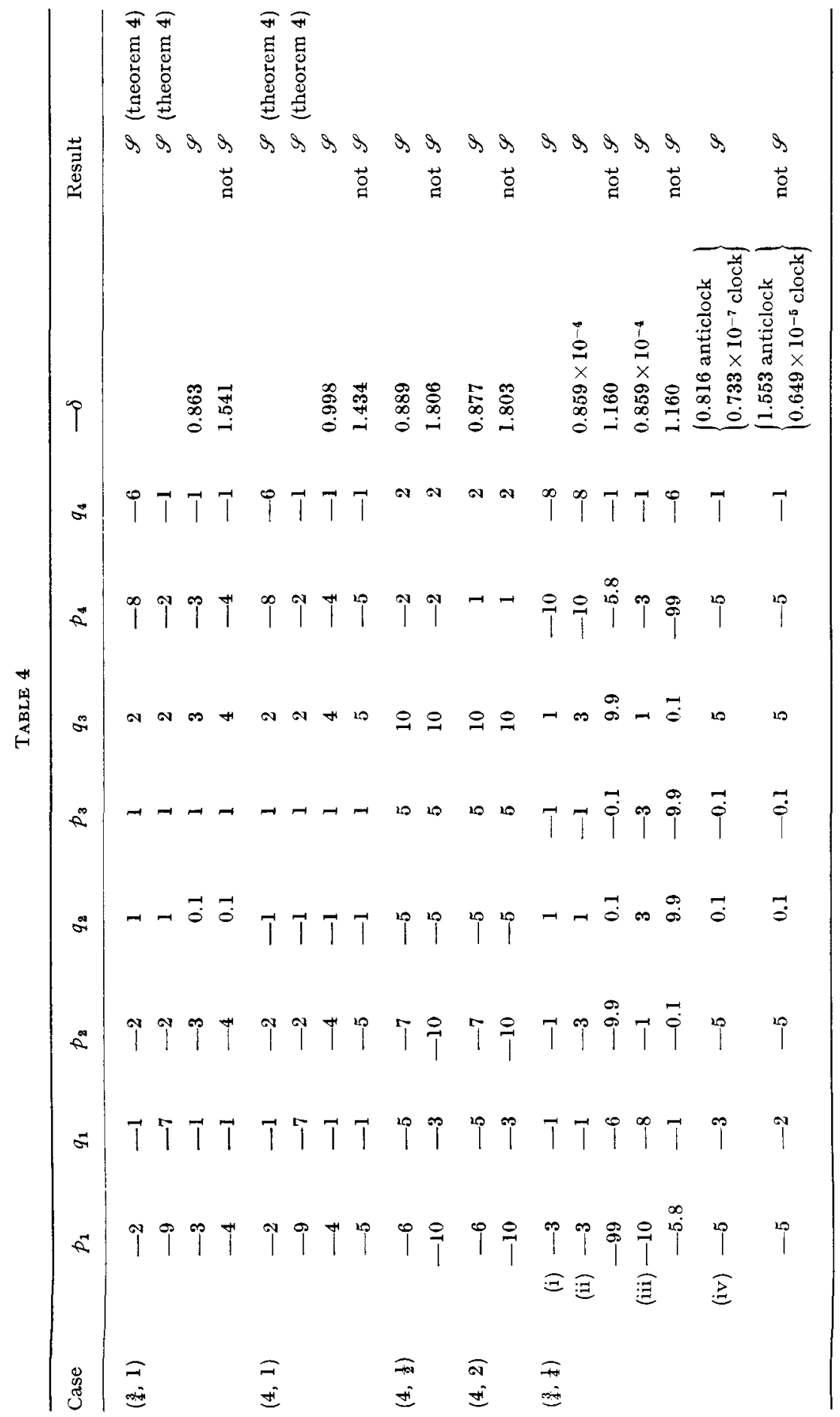




\section{References}

[1] D. E. Daykin, H. T. Loh, 'Stability of a Pair of First Order Differential Equations with Bounded Coefficients', (1965) (Unpublished).

[2] D. E. Daykin, K. W. Chang, 'On the Stability of a Second Order Differential Equation', Jour. Australian Math. Soc., 5 (1965) 8-16.

University of Malaya

Kuala Lumpur 